OPEN ACCESS

Edited by:

Fenglin Liu,

Fudan University, China

Reviewed by:

Xin-Zu Chen,

Sichuan University, China

Zhang Heng,

Fudan University, China

*Correspondence:

Changming Huang hcmlr2002@163.com

Chaohui Zheng

wwkzch@163.com

Ping Li

24627878@qq.com

${ }^{\dagger}$ These authors have contributed equally to this work

Specialty section: This article was submitted to Gastrointestinal Cancers, a section of the journa

Frontiers in Oncology

Received: 14 January 2020 Accepted: 15 December 2020 Published: 11 February 2021

Citation:

Lu J, Xu B, Xu Y, Wu Y, Xie J, Wang J, Lin J, Chen Q, Cao L, Zheng C,

Huang C and Li P (2021) A Novel Insight Into Fecal Occult Blood Test for the Management of Gastric Cancer: Complication,

Survival, and Chemotherapy Benefit After Ro Resection.

Front. Oncol. 10:526746. doi: 10.3389/fonc.2020.526746

\section{A Novel Insight Into Fecal Occult Blood Test for the Management of Gastric Cancer: Complication, Survival, and Chemotherapy Benefit After R0 Resection}

Jun $L u^{1,2,3 t}$, Binbin $X u^{1,2,3 t}, Y u X u^{4 t}$, Yuan Wu ${ }^{4}$, Jianwei Xie ${ }^{1,2,3}$, Jiabin Wang ${ }^{1,2,3}$, Jianxian Lin ${ }^{1,2,3}$, Qiyue Chen ${ }^{1,2,3}$, Longlong Cao ${ }^{1,2,3}$, Chaohui Zheng ${ }^{1,2,3 *}$, Changming Huang ${ }^{1,2,3^{*}}$ and Ping $\mathrm{Li}^{1,2,3^{*}}$

${ }^{1}$ Department of Gastric Surgery, Fujian Medical University Union Hospital, Fuzhou, China, ${ }^{2}$ Department of General Surgery, Fujian Medical University Union Hospital, Fuzhou, China, ${ }^{3}$ Key Laboratory of Ministry of Education of Gastrointestinal Cancer, Fujian Medical University, Fuzhou, China, ${ }^{4}$ Department of Pathology, the School of Basic Medical Sciences, Fujian Medical University, Fuzhou, China

Background: Previous studies have shown that the all-cause mortality and noncolorectal cancer mortality of patients with fecal occult blood test (FOBT) positivity are significantly increased, implying that FOBT results may have more prognostic value.

Methods: Retrospective analysis was performed for gastric cancer (GC) patients who underwent RO gastrectomy from July 2007 to July 2014 at our hospital. Propensity score matching (PSM) was used to reduce confounding bias and a computerized technique for the nearest available score matching without replacement was applied. The cumulative survival rate was calculated using the Kaplan-Meier method and a log-rank test. Cox proportional hazards regression and logistic regression was used to determine the independent prognostic factors associated with survival and postoperative complications, respectively. The expression level of tumor-associated macrophages (TAMs) and proinflammatory cytokines (TNF- $\alpha$, IL-6) were evaluated by immunohistochemical $(\mathrm{IHC})$.

Results: A total of 3,003 patients were included and 246 patients (8.2\%) were in preoperative FOBT positive status. There was no significant difference in demographic data between preoperative FOBT positive and negative group after a 1:4 PSM. The overall postoperative complications, major complications, and anastomotic leakage were significantly higher in the preoperative FOBT-positive group than in the preoperative FOBT-negative group. Moreover, preoperative FOBT-positivity was an independent risk factor for 5-year overall survival (OS) (HR: 1.32, $\mathrm{p}=0.005)$. For stage II/III patients, the postoperative adjuvant chemotherapy (PAC) benefit was found in preoperative FOBTnegative group (5-year OS: 49.9 vs. 36.8\%, $p=0.001$ ), whereas the PAC benefit was lost in preoperative FOBT-positive groups (5-year OS: 40.8 vs. $37.7 \% p=0.896$ ). Finally, $I H C$ found that preoperative FOBT-positivity in patients was significantly associated with 
higher TAMs infiltration and higher expression of IL-6 and TNF- $\alpha$ in tumor tissues than in the preoperative FOBT-negative group.

Conclusion: As a simple and low-cost method, preoperative FOBT results can predict both complications and survival after R0 gastrectomy for GC. More importantly, stage II/III GC patients with FOBT-positive seem not benefit from PAC alone. Further exploration is warranted.

Keywords: gastric cancer, fecal occult blood test, adjuvant chemotherapy, prognosis, tumor immune microenvironment

\section{INTRODUCTION}

In the past few decades, despite significant advances in early diagnosis, radical surgery, and chemotherapy, gastric cancer (GC) remains the fifth-most common malignancy in the world and ranks third in tumor-related mortality (1). Radical gastrectomy is the dominant treatment for patients with resectable gastric cancer. Looking for indicators that can effectively predict complications and prognosis in GC patients may help develop individualized treatment options to improve patients' outcomes.

In addition, there are large differences in the postoperative adjuvant chemotherapy (PAC) response. Increasing numbers of scholars have explored the predictive indicators of chemotherapy response. It has been found that some prognostic scores based on the tumor immune microenvironment (TIM) or gene expression predicted the PAC response in stage II/III GC patients (2-7). However, they have not been routinely used in clinical practice due to their complicated operation and high cost.

The fecal occult blood test (FOBT) has been widely used for screening colorectal cancer (8). Recently, several studies have shown that people with FOBT-positivity have a significantly higher mortality rate, including colorectal and non-colorectal cancer mortality, than those with FOBT-negativity $(9,10)$, suggesting that FOBT results can become potential population prognostic indicators, especially for cancer patients. However, there are no studies reporting the value of the FOBT in predicting the short-term and long-term effects of R0 after GC patients and the benefits of PAC.

Therefore, the present study aimed to explore the impact of the FOBT results on the long-term prognosis of and PAC benefit in GC patients after radical resection. In addition, we analyzed the relationship between FOBT status and the local tumor immune microenvironment (TIM) by immunohistochemistry (IHC) analysis to speculate the potential molecular mechanism underlying how the FOBT results affects clinical efficacy in the treatment of GC.

\section{MATERIALS AND METHODS}

\section{Study Population}

In this retrospective analysis, data were collected from 3,343 patients diagnosed with primary gastric adenocarcinoma at Department of Gastric Surgery, Fujian Medical University
Union Hospital (FMUUH) from July 2007 to July 2014. Two attending physicians staged the tumor before the operation according to gastroscopy, total abdominal CT and enhancement, total abdominal ultrasonography, and other examination results (11). Digital rectal examination and total abdominal CT were performed to preliminarily determine whether the patient has a colorectal tumor. Colonoscopy is performed only when the patient is suspected of having a colorectal tumor by total abdominal CT. The exclusion criteria were as follows: 1) patients with a history of other malignant tumors within 5 years $(\mathrm{n}=18)$; 2$)$ neoadjuvant chemotherapy $(\mathrm{n}=89)$; 3) intraperitoneal or distant metastasis confirmed during or after the operation $(n=103)$; 4) gastric stump cancer $(n=99)$; and 5) unavailable data of the FOBT results $(n=31)$. A total of 340 patients were excluded. The remaining 3003 patients undergoing radical gastrectomy were entered into the statistical analysis (see flowchart in Supplementary Figure S1). Postoperative pathological TNM (pTNM) staging was based on the 7th American Joint Committee on Cancer (AJCC) staging system. Patients in stage I were excluded from a subset analysis assessing the benefits of PAC. Written informed consent was obtained from all patients before sample collection, and the study procedures were approved by the Institutional Review Board of Fujian Medical University Union Hospital.

\section{Fecal Occult Blood Test}

Preoperative stool routine and FOBT were administered to all patients at our institution, unless patients' refusal or an inappropriate physical condition. The fecal occult blood test kit was obtained from Baso Diagnostics, China, with a detection limitation of $50 \mu \mathrm{g}(\mathrm{Hb}) / \mathrm{g}$ (stool), as previously described (12). The FOBT was usually conducted within 1 week before surgery.

\section{Immunohistochemistry}

Formalin-fixed, paraffin-embedded GC surgical specimens, obtained from 120 GC patients, were used for immunohistochemistry (IHC). The contents of infiltrated macrophages, interleukin-6 (IL-6), and tumor necrosis factor- $\alpha$ (TNF- $\alpha$ ) in the individual GC specimens were characterized by IHC using an avidin-biotin peroxidase complex method, as previously reported (13). Briefly, Slides (4- $\mu \mathrm{m}$ thick consecutive paraffin sections) from the blocks with the highest tumor content for each sample were used for immunohistochemical staining and immersed in xylene and rehydrated through graded concentrations 
of ethanol followed by PBS buffer and deionized water for 5 min each. Slides were then heated to $100^{\circ} \mathrm{C}$ for $20 \mathrm{~min}$ in a $\mathrm{pH} 9$ Trisbased solution. All slides were incubated with the primary antibodies for $60 \mathrm{~min}$ at $37^{\circ} \mathrm{C}$ for $1 \mathrm{~h}$ (dilutions: mouse antiCD68 1:500, Maixin Bio, Fuzhou, China; rabbit anti-human TNF alpha 1:300, Abcam ab6671, Cambridge, UK; mouse anti-human IL-6 1:400, Abcam ab9324, Cambridge, UK) and were then washed. A secondary antibody for mouse IgG was added for $30 \mathrm{~min}$ and the slides were again washed. The sections were processed with the universal SP Elivision-Plus Kit (Maixin Bio, Fuzhou, China). Finally, the sections were counterstained with hematoxylin.

\section{Evaluation of Immunohistochemical Staining}

Individual specimens were evaluated by two pathologists (Xu Y and $\mathrm{Wu} \mathrm{Y}$ ) in a blind manner. CD68+ TAMs were estimated by counting the number of CD68+ TAMs in each of the 3 tissue cores from each patient tumor sample, and the mean of 3 counts was recorded. The percentages of $\mathrm{CD} 68+$ cells in three representative high power fields of individual samples were analyzed for macrophage infiltration and were scored as 0 ( $<5 \%$ of CD $68+$ cells), 1 (5-25\%), $2(>25-50 \%)$, or $3(>50 \%)$, as previously described (14). A score of 0 or 1 with anti-CD68 on immunohistochemistry was regarded as "low TAM infiltration" and 2 or 3 staining as "high TAM infiltration" (14).

IL- 6 or TNF- $\alpha$ positivity was scored 0 to 3 as a proportion of tumor cells as follows: $<5 \%=0 ; 5 \%$ to $25 \%=1 ; 26 \%$ to $50 \%=2$; or $>50 \%=3$. Staining intensity was also scored as negative $(0)$, weakly positive (1), moderately positive (2), or strongly positive (3). Based on the sum of these two scores, patients were then dichotomized into "low expression" (score of 0-2) and "high expression" (score of 3-6) groups (15). (Supplementary Figures S2-S4).

\section{Propensity Score Matching}

The propensity scores were calculated using a logistic regression model, and the following covariates were included: age, sex, the Charlson Comorbidity Index (CCI), American Society of Anesthesiologists (ASA) grade, adjuvant chemotherapy, pT, $\mathrm{pN}$, hemoglobin $(\mathrm{Hb})$ level, and albumin (Alb) level. We used a computerized technique for the nearest available score matching without replacement (11) using SPSS 18.0 (SPSS Inc., Chicago, IL, USA).

\section{Complications}

Complications were defined as previously described (11). Postoperative complications were graded according to the Clavien-Dindo classification system; complications greater than grade III were defined as serious complications (16).

\section{Surgery}

In the present study, all the patients had undergone D2 radical gastrectomy with the same group of surgeons. The following lymphadenectomy sequences were performed: 1) for distal gastrectomy, no. $6 \rightarrow$ no. $7,9,11 \mathrm{p} \rightarrow$ no. $3,1 \rightarrow$ no. $8 \mathrm{a}, 12 \mathrm{a}$, $5 \rightarrow$ no. $4 \mathrm{sb}$; and for 2 ) total gastrectomy: no. $6 \rightarrow$ no.7, $9,11 \mathrm{p} \rightarrow$ no. 8 a, 12 a, $5 \rightarrow$ no. $1 \rightarrow$ no. $4 \mathrm{sb} \rightarrow$ no. $10,11 d \rightarrow$ no. 2 . The additional details were described in the previous study (17-20).

\section{Adjuvant Chemotherapy}

According to the patient's wishes and their physical condition, fluoride-based adjuvant chemotherapy was recommended for most patients with pathological stage II and III disease in our center, as previously described (21). Final decision to administer adjuvant chemotherapy was made after careful discussion between the clinician and the patients.

\section{Follow-Up}

The median follow-up time was 72 months (95\% CI 71-74 months). Overall survival (OS) was defined as the period from the date of surgery to the date of death or the final follow-up (11). Postoperative follow-ups were performed every 3 months for 2 years and then every 6 months from years 3 to 5 . Most routine patient follow-up appointments included a physical examination, laboratory tests, chest radiography, abdominal ultrasonography or CT and an annual endoscopic examination (11).

\section{Statistical Analysis}

Continuous variables are reported as the means \pm SD. Categorical and continuous variables were compared using a $\chi 2$ test or Fisher's exact test and a t test, respectively. The cumulative survival rate was calculated using the KaplanMeier method and a log-rank test. A Cox proportional hazards regression model was used to determine the independent prognostic factors associated with survival. All statistical analyses were performed using SPSS v.18.0 for Windows (SPSS Inc., Chicago, IL, USA) and R (https:// www.r-project.org/). Values of p lower than 0.05 were considered statistically significant.

\section{RESULTS}

\section{Clinicopathological Characteristics of the Patients}

Table 1 shows the demographic data of all the patients $(n=$ $3,003)$ and the propensity score-matched patients $(n=1,230)$. A total of 246 patients $(8.2 \%)$ were FOBT-positive with a worse preoperative status (e.g., age, ASA score, $\mathrm{CCI}, \mathrm{Hb}, \mathrm{Alb}$ ), and the tumor stage was more advanced (all $\mathrm{p}<0.05)$. In the FOBT $(+)$ group, patients with the atrial fibrillation $(1.2 \%)$, coronary heart disease $(3.3 \%)$, peptic ulcer $(1.2 \%)$, or inflammatory bowel disease $(0 \%)$ only occupied a smaller proportion. After propensity score matching (PSM) for $1: 4$ to eliminate the baseline bias, no significant differences between the FOBTpositive group $(\mathrm{n}=246)$ and the FOBT-negative group ( $\mathrm{n}=$ 984) were observed in clinicopathological characteristics (all $\mathrm{p}>0.05$ ).

\section{Perioperative Outcome After Surgery}

The mean operative time and intraoperative blood loss were comparable between the FOBT-positive group and the FOBT- 
TABLE 1 | Clinicopathological characteristics of patients before and after matching.

\begin{tabular}{|c|c|c|c|c|c|c|}
\hline & \multicolumn{3}{|c|}{ All patients } & \multicolumn{3}{|c|}{ Propensity-matched patients } \\
\hline & FOBT $(-)(n=2,757)$ & FOBT $(+)(n=246)$ & $p$ value & FOBT (-) (n = 984) & FOBT $(+)(n=246)$ & $p$ value \\
\hline Age & & & $<0.001$ & & & 0.819 \\
\hline$<65$ & $1,734(62.9 \%)$ & 127 (51.6\%) & & $516(52.4 \%)$ & 127 (51.6\%) & \\
\hline$\geq 65$ & $1,023(37.1 \%)$ & 119 (48.4\%) & & $468(47.6 \%)$ & 119 (48.4\%) & \\
\hline Sex n (\%) & & & 0.026 & & & 0.526 \\
\hline Female & 704 (25.5\%) & $47(19.1 \%)$ & & $206(20.9 \%)$ & 47 (19.1\%) & \\
\hline Male & $2,053(74.5 \%)$ & 199 (80.9\%) & & $778(79.1 \%)$ & 199 (80.9\%) & \\
\hline Charlson Comorbidity Index, n (\%) & & & 0.005 & & & 0.509 \\
\hline 0 & $1,908(69.2 \%)$ & $154(62.6 \%)$ & & 607 (61.7\%) & $154(62.6 \%)$ & \\
\hline 1 & $628(22.8 \%)$ & $58(23.6 \%)$ & & $261(26.5 \%)$ & $58(23.6 \%)$ & \\
\hline$\geq 2$ & 221 (8.0\%) & $34(13.8 \%)$ & & $116(11.8 \%)$ & 34 (13.8\%) & \\
\hline \multicolumn{7}{|l|}{ Comorbidity, n (\%) } \\
\hline Atrial fibrillation & $14(0.5 \%)$ & $3(1.2 \%)$ & 0.158 & $5(0.5 \%)$ & $3(1.2 \%)$ & 0.369 \\
\hline Coronary heart disease & 107 (3.9\%) & $8(3.3 \%)$ & 0.167 & $52(5.3 \%)$ & $8(3.3 \%)$ & 0.186 \\
\hline Peptic ulcer & $21(0.8 \%)$ & $3(1.2 \%)$ & 0.441 & $6(0.5 \%)$ & $3(1.2 \%)$ & 0.369 \\
\hline IBD & $1(0.04 \%)$ & $1(0.4 \%)$ & 0.157 & $0(0.0 \%)$ & $1(0.4 \%)$ & 0.200 \\
\hline ASA & & & 0.020 & & & 1.000 \\
\hline$<3$ & 2,626 (95.2\%) & 226 (91.9\%) & & 904 (91.9\%) & 226 (91.9\%) & \\
\hline$\geq 3$ & 131 (4.8\%) & 20 (8.1\%) & & $80(8.1 \%)$ & 20 (8.1\%) & \\
\hline $\mathrm{BMl}$ & & & 0.428 & & & 0.774 \\
\hline$<25$ & 2,335 (84.7\%) & $213(86.6 \%)$ & & 845 (85.9\%) & $213(86.6 \%)$ & \\
\hline$\geq 25$ & 422 (15.3\%) & $33(13.4 \%)$ & & $139(14.1 \%)$ & 33 (13.4\%) & \\
\hline Tumor size n (\%) & & & 0.209 & & & 0.253 \\
\hline$<50$ mm & $1,471(53.4 \%)$ & $121(49.2 \%)$ & & $524(53.3 \%)$ & $121(49.2 \%)$ & \\
\hline$\geq 50 \mathrm{~mm}$ & $1,286(46.6 \%)$ & 125 (50.8\%) & & $460(46.7 \%)$ & 125 (50.8\%) & \\
\hline Tumor location n (\%) & & & 0.568 & & & 0.744 \\
\hline Upper & $723(26.2 \%)$ & $62(25.2 \%)$ & & 281 (28.6\%) & $62(25.2 \%)$ & \\
\hline Middle & 552 (20.0\%) & 49 (19.9\%) & & $188(19.1 \%)$ & 49 (19.9\%) & \\
\hline Lower & $1,182(42.9 \%)$ & $101(41.1 \%)$ & & 393 (39.9\%) & $101(41.1 \%)$ & \\
\hline Mix & 300 (10.9\%) & $34(13.8 \%)$ & & $122(12.4 \%)$ & $34(13.8 \%)$ & \\
\hline Gastrectomy extent n (\%) & & & 0.350 & & & 0.350 \\
\hline Distal & $1,437(52.1 \%)$ & 139 (56.5\%) & & $1,437(52.1 \%)$ & 139 (56.5\%) & \\
\hline Total & $1,263(45.8 \%)$ & $101(41.1 \%)$ & & $1,263(45.8 \%)$ & $101(41.1 \%)$ & \\
\hline Others & $57(2.1 \%)$ & $6(2.4 \%)$ & & $57(2.1 \%)$ & $6(2.4 \%)$ & \\
\hline Reconstruction & & & 0.031 & & & 0.596 \\
\hline B-I & 938 (34.0\%) & 75 (30.5\%) & & 307 (31.2\%) & 75 (30.5\%) & \\
\hline B-II & 297 (10.8\%) & $18(7.3 \%)$ & & $83(8.4 \%)$ & $18(7.3 \%)$ & \\
\hline Roux-en-Y & $1,437(52.1 \%)$ & $139(56.5 \%)$ & & $556(56.5 \%)$ & $139(56.5 \%)$ & \\
\hline Others & $85(3.1 \%)$ & $14(5.7 \%)$ & & 38 (3.9\%) & $14(5.7 \%)$ & \\
\hline Histologic type n (\%) & & & 0.976 & & & 0.863 \\
\hline Well & $136(4.9 \%)$ & 12 (4.9\%) & & 41 (4.2\%) & 12 (4.9\%) & \\
\hline Moderate & $982(35.6 \%)$ & $86(35.0 \%)$ & & 339 (34.5\%) & $86(35.0 \%)$ & \\
\hline Poor & 1,639 (59.4\%) & $148(60.2 \%)$ & & $553(56.2 \%)$ & $148(60.2 \%)$ & \\
\hline Lymphovascular invasion n (\%) & & & 0.314 & & & 0.741 \\
\hline Absent & $2,014(73.1 \%)$ & 187 (76.0\%) & & $738(75.0 \%)$ & 187 (76.0\%) & \\
\hline Present & $743(26.9 \%)$ & 59 (24.0\%) & & $246(25.0 \%)$ & $59(24.0 \%)$ & \\
\hline Adjuvant chemotherapy n (\%) & & & $<0.001$ & & & 0.819 \\
\hline Absent & $1,596(57.9 \%)$ & $111(45.1 \%)$ & & 452 (45.9\%) & $111(45.1 \%)$ & \\
\hline Present & $1,161(42.1 \%)$ & 135 (54.9\%) & & $532(54.1 \%)$ & 135 (54.9\%) & \\
\hline pT stage n (\%) & & & $<0.001$ & & & 0.253 \\
\hline $\mathrm{T} 1$ & 691 (25.1\%) & $41(16.7 \%)$ & & 142 (14.4\%) & 41 (16.7\%) & \\
\hline T2 & 337 (12.2\%) & 30 (12.2\%) & & 103 (10.5\%) & 30 (12.2\%) & \\
\hline Т3 & 759 (27.5\%) & $55(22.4 \%)$ & & $280(28.5 \%)$ & $55(22.4 \%)$ & \\
\hline $\mathrm{T} 4$ & 970 (35.2\%) & 120 (48.8\%) & & 459 (46.6\%) & 120 (48.8\%) & \\
\hline pN stage n (\%) & & & $<0.001$ & & & 0.917 \\
\hline NO & 1,060 (38.4\%) & 67 (27.2\%) & & 252 (25.6\%) & 67 (27.2\%) & \\
\hline N1 & $414(15.0 \%)$ & $36(14.6 \%)$ & & $138(14.0 \%)$ & $36(14.6 \%)$ & \\
\hline N2 & $444(16.1 \%)$ & 43 (17.5\%) & & 171 (17.4\%) & 43 (17.5\%) & \\
\hline N3 & 839 (30.4\%) & 100 (40.7\%) & & 423 (43.0\%) & 100 (40.7\%) & \\
\hline pTNM stage n (\%) & & & $<0.001$ & & & 0.861 \\
\hline 1 & $830(30.1 \%)$ & 49 (19.9\%) & & $184(18.7 \%)$ & 49 (19.9\%) & \\
\hline$\|$ & $618(22.4 \%)$ & 49 (19.9\%) & & 190 (19.3\%) & 49 (19.9\%) & \\
\hline
\end{tabular}




\begin{tabular}{|c|c|c|c|c|c|c|}
\hline & \multicolumn{3}{|c|}{ All patients } & \multicolumn{3}{|c|}{ Propensity-matched patients } \\
\hline III & $1,309(47.5 \%)$ & $148(60.2 \%)$ & & $610(62.0 \%)$ & $148(60.2 \%)$ & \\
\hline$<90 \mathrm{~g} / \mathrm{L}$ & $276(10.0 \%)$ & 69 (28.0\%) & & $232(23.6 \%)$ & 69 (28.0\%) & \\
\hline$\geq 90 \mathrm{~g} / \mathrm{L}$ & 2,481 (90.0\%) & $177(72.0 \%)$ & & $752(76.4 \%)$ & $177(72.0 \%)$ & \\
\hline Albumin n (\%) & & & $<0.001$ & & & 0.529 \\
\hline Death n (\%) & & & $<0.001$ & & & 0.001 \\
\hline no & $1,716(62.2 \%)$ & $111(45.1 \%)$ & & $561(57.0 \%)$ & $111(45.1 \%)$ & \\
\hline yes & 1,041 (37.8\%) & 135 (54.9\%) & & 423 (43.0\%) & 135 (54.9\%) & \\
\hline
\end{tabular}

FOBT indicates fecal occult blood test; ASA, American Society of Anesthesiologists; BMI, body mass index; IBD, inflammatory bowel disease.

negative group $(216.58 \pm 72.72 \mathrm{~min} v s .213 .82 \pm 72.24 \mathrm{~min}, \mathrm{p}=$ $0.592 ; 124.22 \pm 177.43 \mathrm{ml} v s .117 .04 \pm 132.84 \mathrm{ml}, \mathrm{p}=0.483$ respectively). In addition, from the perspective of postoperative recovery, there were no significant differences in the time to flatus ( $3.85 \pm 1.35$ days $v s .3 .72 \pm 1.22$ days, $\mathrm{p}=0.099)$, the time to food intake $(5.12 \pm 1.99$ days $v s .4 .90 \pm 1.61$ days, $\mathrm{p}=0.463)$ and the postoperative hospital stays ( $15.47 \pm 8.32$ days $v s .15 .02 \pm$ 8.66 days, $\mathrm{p}=0.150$ ) (Table 2 ).

\section{Postoperative Complications}

Overall, postoperative complications occurred in 50 (20.3\%) and $146(14.8 \%)$ patients in the FOBT-positive and FOBT-negative groups $(\mathrm{p}=0.035)$, respectively. Among the surgical complications, the incidence of anastomotic fistula was significantly higher in the FOBT-positive group than in the FOBT-negative group [9 (3.7\%) vs. 10 (1.0\%), p = 0.003]. Postoperative ileus, abdominal bleeding, wound infection and abdominal infection were not significantly different between the two groups. Among the non-surgical complications, there were no significant differences in postoperative pneumonia or disorders of the cardiovascular, liver, and urinary systems between the two groups. According to the Clavien-Dindo classification, the incidence of major complications in the FOBT-positive group was significantly higher than that in the FOBT-negative group [15 (6.1\%) vs. 33 (3.4\%), p = 0.047]. In terms of postoperative complications, there was no significant difference in the time of postoperative complications between the FOBT-positive patients and negative patients (6.60 \pm 4.80 days vs. $5.66 \pm 3.44$ days, respectively, $\mathrm{p}=0.205)$.

\section{Univariable and Multivariate Analyses of Factors Associated With Overall Survival}

In the univariate analysis, age $\geq 65, \mathrm{BMI} \geq 25$, FOBT-positive, histologic type, tumor location, lymphovascular invasion, pTNM stage, preoperative $\mathrm{Hb}<90 \mathrm{~g} / \mathrm{L}$, and preoperative $\mathrm{Alb}<35 \mathrm{~g} / \mathrm{L}$ were closely related to overall survival, all $\mathrm{p}<0.05$. In the multivariate analysis, age $\geq 65$, FOBT-positive, pTNM stage, and preoperative $\mathrm{Alb}<35 \mathrm{~g} / \mathrm{L}$ were independent prognostic factors affecting long-term survival, all $\mathrm{p}<0.05$ (Table 3).

TABLE 2 | Perioperative outcome and postoperative complication after surgery.

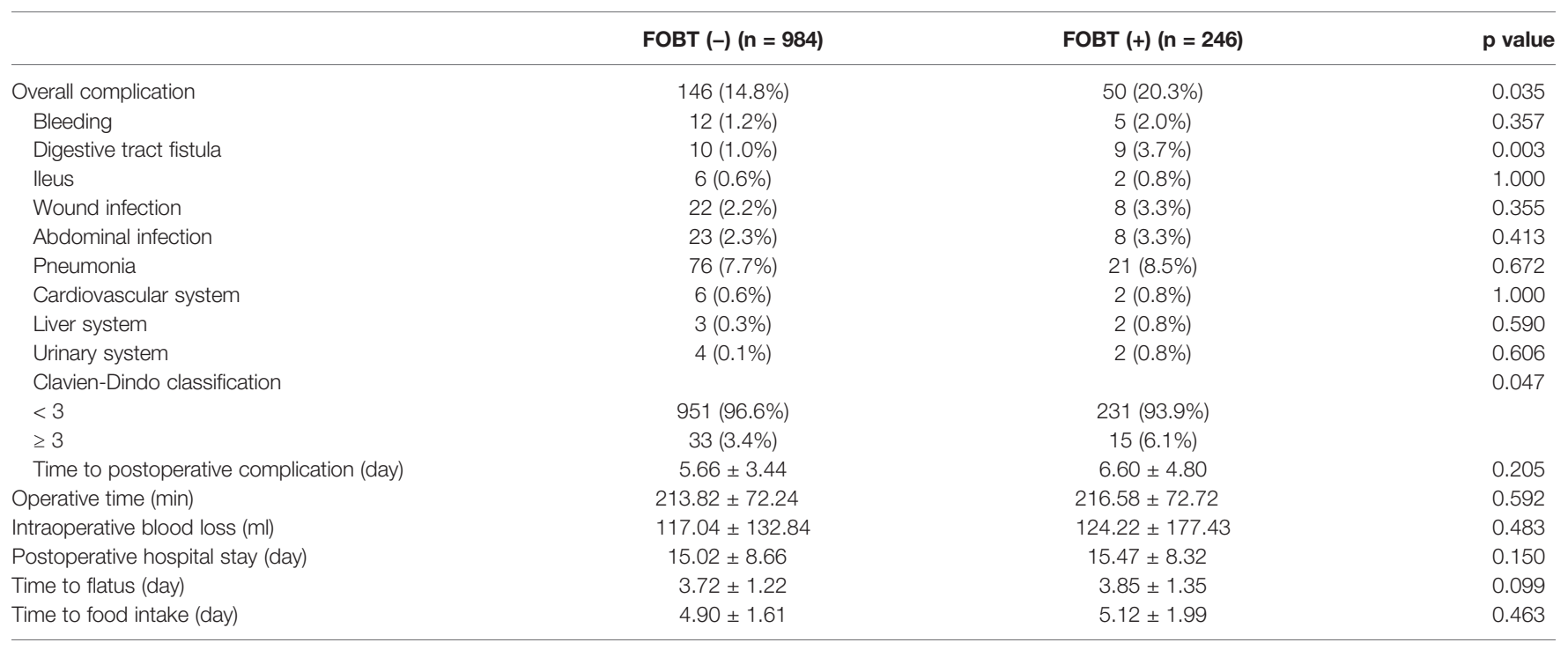

FOBT indicates fecal occult blood test. 
TABLE 3 | Univariate and multivariate analyses of factors associated with overall survival.

\begin{tabular}{|c|c|c|c|c|c|c|}
\hline & \multicolumn{3}{|c|}{ Univariate analysis } & \multicolumn{3}{|c|}{ Multivariate analysis } \\
\hline & HR & $95 \% \mathrm{Cl}$ & $p$ value & HR & $95 \% \mathrm{Cl}$ & $p$ value \\
\hline Age $\geq 65$ vs. $<65$ & 1.543 & $1.305-1.824$ & $<0.001$ & 1.367 & $1.153-1.620$ & $<0.001$ \\
\hline Male sex vs. female & 1.021 & $0.833-1.252$ & 0.843 & & & \\
\hline \multicolumn{7}{|l|}{ Charlson comorbidity index } \\
\hline 0 & 1.000 & & & & & \\
\hline 1 & 0.922 & $0.756-1.125$ & 0.424 & & & \\
\hline$\geq 2$ & 1.243 & $0.976-1.582$ & 0.077 & & & \\
\hline$A S A \geq 3$ vs. $<3$ & 1.149 & $0.864-1.529$ & 0.340 & & & \\
\hline $\mathrm{BMI} \geq 25$ vs. $<25$ & 0.720 & $0.555-0.934$ & 0.013 & 0.840 & $0.647-1.091$ & 0.191 \\
\hline FOBT positive vs. negative & 1.305 & $1.075-1.584$ & 0.007 & 1.320 & $1.085-1.606$ & 0.005 \\
\hline Tumor size $\geq 50$ vs. $<50$ mm & 1.027 & $0.870-1.213$ & 0.751 & & & \\
\hline \multicolumn{7}{|l|}{ Tumor location } \\
\hline Lower & 1.000 & & & 1.000 & & \\
\hline Middle & 1.180 & $0.933-1.493$ & 0.166 & 1.036 & $0.818-1.314$ & 0.769 \\
\hline Upper & 1.229 & $0.999-1.512$ & 0.051 & 1.141 & $0.926-1.406$ & 0.215 \\
\hline Mix & 1.682 & $1.311-2.158$ & $<0.001$ & 1.226 & $0.952-1.582$ & 0.114 \\
\hline \multicolumn{7}{|l|}{ Histologic type } \\
\hline Differentiated & 1.000 & & & 1.000 & & \\
\hline Undifferentiated & 1.334 & $1.126-1.581$ & 0.001 & 1.088 & $0.914-1.295$ & 0.344 \\
\hline \multicolumn{7}{|l|}{ Lymphovascular invasion } \\
\hline Absent & 1.000 & & & 1.000 & & \\
\hline Present & 1.391 & $1.167-1.658$ & $<0.001$ & 1.077 & $0.899-1.289$ & 0.423 \\
\hline \multicolumn{7}{|l|}{ pTNM stage } \\
\hline 1 & 1.000 & & & 1.000 & & \\
\hline$\|$ & 3.413 & $2.137-5.449$ & $<0.001$ & 2.894 & $1.807-4.635$ & $<0.001$ \\
\hline III & 9.145 & $6.103-13.908$ & $<0.001$ & 7.645 & $4.998-11.694$ & $<0.001$ \\
\hline Hemoglobin $\geq 90$ vs. $<90 \mathrm{~g} / \mathrm{L}$ & 0.684 & $0.570-0.822$ & $<0.001$ & 0.899 & $0.739-1.094$ & 0.288 \\
\hline Albumin $\geq 35$ vs. $<35 \mathrm{~g} / \mathrm{L}$ & 0.522 & $0.442-0.618$ & $<0.001$ & 0.663 & $0.553-0.795$ & $<0.001$ \\
\hline
\end{tabular}

FOBT indicates fecal occult blood test; ASA, American Society of Anesthesiologists; BMI, body mass index.

\section{Overall Survival and Subgroup Analysis}

Kaplan-Meier curve analysis showed that the prognosis of the FOBT-positive group was significantly worse than that of the FOBT-negative group (5-year OS: $48.2 v s .58 .8 \%$, respectively, $\mathrm{p}=0.007)$. Stratified analysis showed that the OS rate of the FOBT-positive group was significantly lower than that of the FOBT-negative group at all pathological stages (5-year OS: p stage I: 82.7 vs. $92.1 \%, \mathrm{p}=0.040$; p stage II: 58.2 vs. $75.3 \%$, $\mathrm{p}=0.039$; $\mathrm{p}$ stage III: 33.5 vs. $43.3 \%, \mathrm{p}=0.036)$ (Figures 1A-D). The same survival results were observed in the early GC group, advanced GC group, lymph node-negative group and lymph node-positive group (Supplementary Figures S5A-D). In addition, in a separate analysis of each clinicopathological factor, the prognostic value of the FOBT result was consistent, including age, tumor site, histologic, and so on (Supplementary Figure S6).

\section{Effects of FOBT Results on Adjuvant Chemotherapy}

Further PAC benefit analysis for stage II/III GC patients after PSM showed that PAC improved the prognosis significantly (5year OS: 52.1 vs. $42.8 \%, \mathrm{p}=0.003$, respectively). Subgroup analysis showed that FOBT-negative patients benefited from PAC (5-year OS: 49.9 vs. $36.8 \%, \mathrm{p}=0.001$ ). However, in the FOBT-positive patients, the prognosis was similar between the chemotherapy group and the non-chemotherapy group (5-year OS: 40.8 vs. $37.7 \%$, respectively, $\mathrm{p}=0.896)$ (Figures 2A-C).

\section{Immunohistochemistry Results of CD68,} IL-6, and TNF- $\alpha$ Expression in Tumors

Paraffin-embedded sections of FOBT-negative and FOBTpositive patients (60 cases each) were randomly selected from the propensity score-matched patients $(n=1,230)$ for IHC analysis to explore the association between FOBT status and the tumor immune microenvironment (CD68, IL-6, and TNF- $\alpha$ expression). The clinicopathological characteristics between the two groups were comparable, showed in Supplementary Table S1. The CD68, IL-6, and TNF- $\alpha$ expression in tumor cells was significantly higher in FOBT-positive patients than in FOBTnegative patients (all $\mathrm{p}<0.05$ ) (Table 4).

\section{DISCUSSION}

In a study of individuals in the Netherlands undergoing screening for colorectal cancer by FOBT, it was found that fewer than $1 \%$ of patients with a positive result from the FOBT to receive a diagnosis of gastric cancers within 3 years (22). In the present study, we found that approximately $8 \%$ of GC patients had a preoperative FOBT-positive status, and FOBT-positivity in patients was associated with a worse clinical background and long-term prognosis. After further analyses with a 1:4 PSM, we found that patients with FOBT-positive tumors had a higher incidence of postoperative complications and a worse long-term prognosis than patients with FOBT-negative tumors. The 

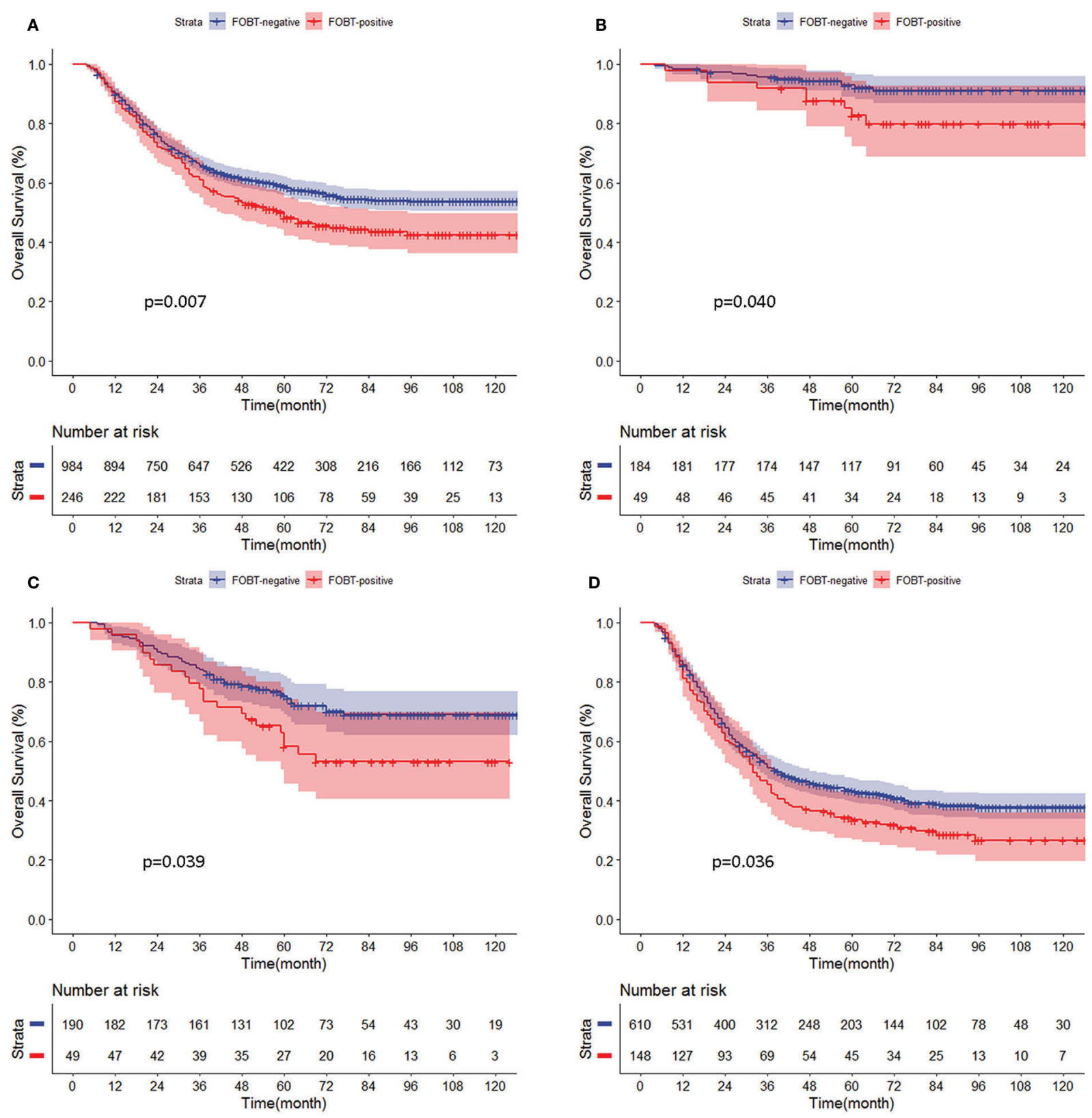

FIGURE 1 | (A-D) Comparison of overall survival curves for propensity score-matched patients between the fecal occult blood test (FOBT)-positive and FOBTnegative groups according to pathological stage. (A) All patients. (B) p stage (I). (C) p stage (II). (D) p stage III.

chemotherapy benefit analysis of stage II/III GC patients found that FOBT-positive patients showed significant chemotherapy resistance. Finally, IHC analysis of tumor tissues in 120 patients showed that the TAM and the expression levels of IL- 6 and TNF$\alpha$ in tumor cells of FOBT-positive patients were significantly higher than those in FOBT-negative patients.

FOBT-positivity is common in digestive tract tumors (gastric cancer, colorectal cancer, etc.), inflammatory bowel disease, peptic ulcers and other diseases $(23,24)$. At present, the FOBT has been widely used in the screening of colorectal cancer in the general population (8). In recent years, studies by Libby and Chen et al. concerning large populations have shown that not only colorectal cancer mortality but also all-cause mortality and non-colorectal cancer mortality are significantly higher in patients who are FOBT-positive than in patients who are FOBT-negative $(9,10)$. It is speculated that the cause for this difference may be related to long-term chronic anemia $(25,26)$ and systemic inflammatory response (9), suggesting that the FOBT results can be a potential predictor of prognosis in a population. However, the relationship between FOBT results and 

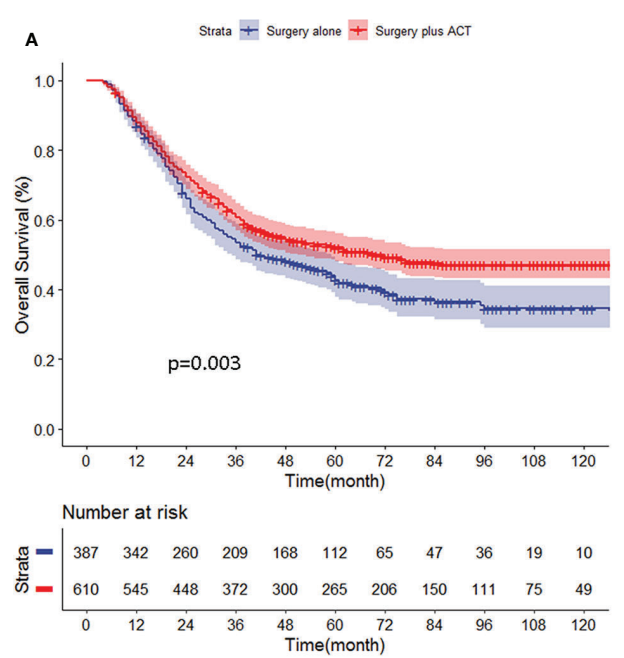

B Strata + Suggery alone $\mp$ Surgery plus ACT
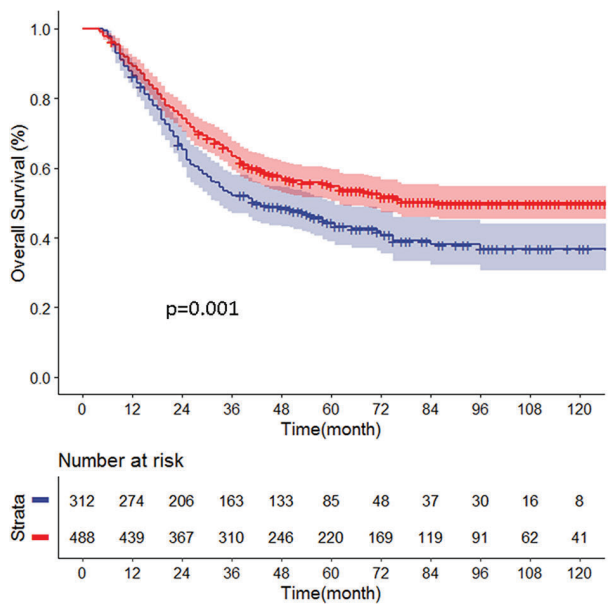

C Strata + Surgery alone $\mp$ Surgery plus ACT

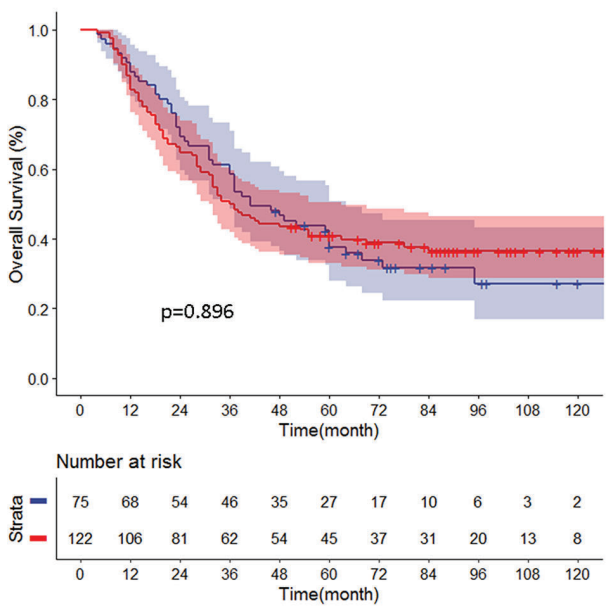

FIGURE 2 | (A-C) Chemotherapy benefit analysis for stage II/III gastric cancer (GC) patients in different groups. (A) All stage II/IIl patients. (B) FOBTnegative patients. (C) FOBT-positive patients. long-term prognosis after radical surgery in GC patients has yet been reported.

Studies have shown that preoperative high CCI (27), low Hb level (28), and low albumin level (29) are associated with a worse prognosis. The present study also found that FOBT-positive patients had worse clinical backgrounds, including higher preoperative $\mathrm{CCI}$, lower $\mathrm{Hb}$, and $\mathrm{Alb}$ than FOBT-negative patients. After PSM, although the two groups had a similar baseline, the prognosis of the FOBT-positive group was significantly worse than that of the FOBT-negative group, and multivariate analysis showed that FOBT-positivity was an independent risk factor for worse prognosis. Therefore, we hypothesized that the mechanism may be related to TIM, postoperative complications, adjuvant chemotherapy resistance, and an imbalance of intestinal microbiota.

Since Virchow first discovered the relationship between inflammation and cancer (30), increasingly more evidence has shown that tumor progression is related not only to the intrinsic properties of the tumor cells but also to the local TIM (2). Tumor cells that highly express TNF- $\alpha$ stimulate M1-type macrophage infiltration and secrete IL-6, which damages mucosal epithelial cells and further causes recessive hemorrhage (14, 31-33). By IHC analysis, we demonstrated that the expression levels of CD68, IL- 6 and TNF- $\alpha$ in tumor cells of FOBT-positive patients were significantly higher than those in FOBT-negative patients. This may explain why patients with positive FOBT had a more advanced tumor and worse prognosis.

In addition, more postoperative overall complications and a high incidence of anastomotic leakage in FOBT-positive patients may also affect prognosis. Similar to the results of our study, previous studies have shown that the occurrence of postoperative complications is closely related to the poor prognosis of GC patients, especially anastomotic leakage $(34,35)$. The mechanism may be related the presence of more significant postoperative inflammation and more severe immunosuppression in GC patients. Infectious complications and sepsis enhance the proinflammatory cytokine cascade, including TNF- $\alpha$, IL-1, IL6 , and IL-8. These immunomodulators affect the function and regulation of natural killer cells, cytotoxic T lymphocytes, and antigen presenting cells $(30,36,37)$. Moreover, postoperative complications can lead to prolonged immunosuppression, which enables residual tumor cells to proliferate and survive in the host for a longer time and promotes the occurrence of micrometastasis (34), which in turn affects the long-term prognosis. In addition, although the cause of postoperative complications associated with FOBT-positivity is not clear, based on the findings of our study, caution should be taken with regard to the potential for postoperative complications in FOBT-positive patients.

Postoperative adjuvant chemotherapy resistance may also affect the long-term prognosis of FOBT-positive GC patients. A large number of studies have confirmed that PAC can significantly improve the prognosis of stage II/III GC patients (38). However, it is also important to identify patients who will clearly benefit from chemotherapy. Zeng and Jiang found that the immune prognosis score based on TIM is related to the 
TABLE 4 | Associations among fecal occult blood test (FOBT) result, CD68, IL-6, and TNF- $\alpha$ expression in tumor cells.

\begin{tabular}{|c|c|c|c|c|c|c|c|c|c|}
\hline & $\begin{array}{c}\text { FOBT (-) } \\
(n=60)\end{array}$ & $\begin{array}{c}\text { FOBT (+) } \\
(n=60)\end{array}$ & $p$ value & $\begin{array}{c}\text { TNF- } \alpha \\
\text { low expression } \\
(n=82)\end{array}$ & $\begin{array}{c}\text { TNF- } \alpha \\
\text { high expression } \\
(n=38)\end{array}$ & $p$ value & $\begin{array}{c}\text { IL-6 } \\
\text { low expression } \\
(n=70)\end{array}$ & $\begin{array}{c}\text { IL-6 } \\
\text { high expression } \\
\text { (n=50) }\end{array}$ & $p$ value \\
\hline CD68 & & & & $<0.001$ & & & $<0.001$ & & $<0.001$ \\
\hline Low expression & 52 (86.7\%) & 34 (56.7\%) & & 70 (85.4\%) & 16 (42.1\%) & & 66 (94.3\%) & 20 (40.0\%) & \\
\hline High expression & $8(13.3 \%)$ & 26 (43.3\%) & & 12 (14.6\%) & 22 (57.9\%) & & $4(5.7 \%)$ & 30 (60.0\%) & \\
\hline IL-6 & & & 0.010 & & & & $<0.001$ & & NA \\
\hline Low expression & 42 (70.0\%) & 28 (46.7\%) & & 60 (73.2\%) & 10 (26.3\%) & & NA & NA & \\
\hline High expression & 18 (30.0\%) & 32 (53.3\%) & & 22 (26.8\%) & 28 (73.7\%) & & NA & NA & \\
\hline TNF- $\alpha$ & & & & $<0.001$ & & NA & & & NA \\
\hline Low expression & 52 (86.7\%) & 30 (50.0\%) & & NA & NA & & NA & NA & \\
\hline High expression & 8 (13.3\%) & 30 (50.0\%) & & NA & NA & & NA & NA & \\
\hline
\end{tabular}

FOBT indicates fecal occult blood test; TNF- $\alpha$, tumor necrosis factor- $\alpha$; IL-6, interleukin-6.

efficacy of PAC in GC patients and speculated that infiltration of lymphocytes in tumor cells indicates a chemotherapy-sensitive phenotype $(2,3)$. In our study, the K-M curves showed that although PAC significantly prolonged OS in the stage II/III group, but stratified analysis showed that FOBT-positive patients did not benefit from PAC. In addition, compared with the chromosomal instability subtypes found by Sohn et al. (4), the single patient classifier constructed by Cheong (5), and the microsatellite instability found by Ji and Young $(6,7)$, the FOBT has a high clinical use rate, is inexpensive and is more accessible. Although the mechanism underlying how FOBT-positivity induces chemotherapy resistance is still unclear, previous studies have shown that TAM and high expression of proinflammatory factors such as TNF- $\alpha$ and IL- 6 are closely related to poor prognosis and chemoresistance $(2,3,32,39-41)$. In the present study, according to the results of the IHC analysis, we speculated that in patients with FOBT-positivity, local TIM (such as TAM, TNF- $\alpha$, IL-6) is conducive to tumor proliferation and vascularization and is associated with adjuvant chemotherapy resistance. Therefore, to further improve the prognosis of FOBT-positive patients, it is necessary to carefully evaluate the potential applications of different adjuvant therapies and immune checkpoint therapy (40) in a well-designed, largescale clinical trial for this particular subgroup of patients.

In addition, a study by Elinav has shown that systemic inflammation can alter the intestinal microbial composition, induce the amplification of genotoxic microorganisms and promote the development of digestive tract tumors (42). Intestinal microbiota can also affect local and systemic inflammation and play an important role in tumor treatment response $(43,44)$. Lida believed that the destruction of intestinal microbiota can reduce the treatment response of subcutaneous tumors in mice to chemotherapy drugs (43). Furthermore, intestinal microflora may also contribute to the formation of anticancer immune responses. The destruction of intestinal microflora can indirectly damage mucosal epithelial cells and negatively impact the prognosis of tumor patients (44). In addition, Japanese scholars found that an increase in the hemoglobin index of gastric mucosa is closely related to Helicobacter pylori (Hp) infection and mucosal inflammatory cell infiltration (45) and is more likely to cause gastric mucosal hemorrhage and FOBT-positivity. It is still unclear whether the occurrence of FOBT-positivity in GC patients and poor prognosis are related to the mucosal damage caused by intestinal bacterial flora imbalance, $\mathrm{Hp}$ infection or inflammatory cell infiltration, and further research is needed in the future to explore its relevance and the corresponding treatment measures (46).

There are many diseases that affect the results of FOBT, such as coronary heart disease, atrial fibrillation, due to a long-term use of anti-coagulation drugs. Intestinal bleeding may also cause by inflammatory bowel disease. However, we found that patients with these diseases only occupied a smaller proportion in the present cohort, and it may have limited influence on the results. In addition, in our center, most patients were diagnosed with GC by gastroscopy biopsy preoperatively, which may make the FOBT positive easily. After a definitive diagnosis, they underwent surgery within a week. No other intervention was applied unless they suffered several symptoms such as stomachache, belch, and so on. Due to the nature of the retrospective study, it's difficult for us to further analyze the relationship between the treatment of these diseases and the results of FOBT. However, the hypothesis you propose is interesting and worth exploring in future research.

The present study has the following limitations: first, it is a single-center retrospective study with a certain bias and without external validation. Second, there were 2,124 patients with stage II/III GC, 1,164 (54.8\%) of which received adjuvant chemotherapy. The proportion of patients with adjuvant chemotherapy in the present study was not high but was similar with previous study $(4,47,48)$. Indeed, it's difficult that all the target patient received adjuvant chemotherapy, which is a limitation and have a certain impact on our study. And we did not analyze the impact of adjuvant chemotherapy cycles on longterm survival. Third, we did not analyze the relationship between the FOBT results and recurrence patterns. Fourth, as an unresolved issue nowadays $(2,3)$, these specimens as well as semi-quantitative IHC evaluations may still not completely reflecting the tumor immune microenvironmental status. Last, because colonoscopy was not routinely performed in our department, we cannot exclude the patients with some intestinal diseases such as inflammatory bowel disease, which had the effect to the FOBT results. Nevertheless, for the first time, the present study used bulk data to report the relationship 
between FOBT results and the short- and long-term outcomes of GC patients after radical surgery and the efficacy of PAC in patients with stage II/III disease. Importantly, we used IHC analysis to speculate on the underlying mechanism. The findings will help clinicians develop the best treatment for FOBT-positive patients and provide direction for further research. Based on the current findings, more attention should be paid to FOBT-positive patients before, during, and after surgery. At the first visit, surgeons should determine if the FOBT-positive patient has any unfavorable clinical background (such as anemia and hypoalbuminemia) that may adversely affect the short-term outcome after gastrectomy and should take appropriate measures to reduce the incidence of early postoperative complications. During follow-up periods, the follow-up should be intensive, and because PAC has no clear effect, combined PAC with immunotherapy should be considered to improve the prognosis of these patients (39, 40, 46).

In summary, although the FOBT has been widely used clinically, its potential prognostic value for cancer patients is rarely reported. The present study demonstrated for the first time that FOBT results have predictive value for postoperative complications and long-term prognosis of GC. In addition, for stage II/III GC patients with FOBT-positive tumors, no significant benefit from PAC alone was observed. The role of FOBT and TAM in chemo-resistance should be explored and further evaluation of macrophage-targeting therapy is warranted. The present study can be used as background data for potential future large-scale, multicenter clinical trials to determine the best treatment decisions for these GC patients after surgery.

\section{DATA AVAILABILITY STATEMENT}

The data sets generated for this study are available on request to the corresponding authors.

\section{ETHICS STATEMENT}

The studies involving human participants were reviewed and approved by the Institutional Review Board of Fujian Medical University Union Hospital. The patients/participants provided their written informed consent to participate in this study.

\section{REFERENCES}

1. Fock KM. Review article: the epidemiology and prevention of gastric cancer. Aliment Pharmacol Ther (2014) 40(3):250-60. doi: 10.1111/apt.12814

2. Jiang Y, Zhang Q, Hu Y, Li T, Yu J, Zhao L, et al. ImmunoScore Signature: A Prognostic and Predictive Tool in Gastric Cancer. Ann Surg (2018) 267 (3):504-13. doi: 10.1097/SLA.0000000000002116

3. Zeng D, Zhou R, Yu Y, Luo Y, Zhang J, Sun H, et al. Gene expression profiles for a prognostic immunoscore in gastric cancer. Br J Surg (2018) 105 (10):1338-48. doi: 10.1002/bjs.10871

\section{AUTHOR CONTRIBUTIONS}

JL, BX, YX, CZ, and $\mathrm{CH}$ conceived of the study, analyzed the data, and drafted the manuscript. YX and YW evaluated the individual specimens. $\mathrm{CZ}, \mathrm{CH}$, and $\mathrm{PL}$ helped critically revise the manuscript for important intellectual content. PL, JX, JW, JXL, QC, and LC helped collect data and design the study. All authors contributed to the article and approved the submitted version.

\section{FUNDING}

This study was funded by the National Nature Science Foundation of China (no. 81871899), Construction Project of Fujian Province Minimally Invasive Medical Center [no. (2017) 171], Natural Science Foundation of Fujian Province (2019J01155), and Fujian provincial science and technology innovation joint fund project plan (2018Y9005) and Middleaged and young backbone talent training project of Fujian Provincial Health Commission (2020GGA038).

\section{SUPPLEMENTARY MATERIAL}

The Supplementary Material for this article can be found online at: https://www.frontiersin.org/articles/10.3389/fonc.2020. 526746/full\#supplementary-material

Supplementary Figure 1 | Flowchart depicting the patient selection process.

Supplementary Figure 2 | Representative pictures of immunohistochemistry (magnification, $\times 200$ ) which showed the different expression grades of macrophage infiltration in tumor cells.

Supplementary Figure $\mathbf{3}$ | Representative pictures of immunohistochemistry (magnification, $\times 200$ ) which showed the different expression grades of IL-6 infiltration in tumor cells.

Supplementary Figure 4 | Representative pictures of immunohistochemistry (magnification, $\times 200$ ) which showed the different expression grades of TNF- $\alpha$ infiltration in tumor cells.

Supplementary Figure 5 | Comparison of overall survival curves for patients between the FOBT-positive and FOBT-negative groups according to $\mathrm{pT}$ and $\mathrm{pN}$ stage. (A) patients with pT1; (B) patients with pT2-4. (C) patients with pNO. (D) patients with $\mathrm{pN}+$.

Supplementary Figure 6 | The relationship between FOBT results and overall survival in various subgroups.

4. Sohn BH, Hwang JE, Jang HJ, Lee HS, Oh SC, Shim JJ, et al. Clinical Significance of Four Molecular Subtypes of Gastric Cancer Identified by The Cancer Genome Atlas Project. Clin Cancer Res (2017). doi: 10.1158/1078-0432.CCR-16-2211

5. Cheong J-H, Yang H-K, Kim H, Kim WH, Kim Y-W, Kook M-C, et al. Predictive test for chemotherapy response in resectable gastric cancer: a multi-cohort, retrospective analysis. Lancet Oncol (2018) 19(5):629-38. doi: 10.1016/S1470-2045(18)30108-6

6. An JY, Kim H, Cheong JH, Hyung WJ, Kim H, Noh SH. Microsatellite instability in sporadic gastric cancer: its prognostic role and guidance for 5FU based chemotherapy after R0 resection. Int J Cancer (2012) 131(2):50511. doi: $10.1002 /$ ijc. 26399 
7. Choi YY, Kim H, Shin SJ, Kim HY, Lee J, Yang HK, et al. Microsatellite Instability and Programmed Cell Death-Ligand 1 Expression in Stage II/III Gastric Cancer: Post Hoc Analysis of the CLASSIC Randomized Controlled study. Ann Surg (2019) 270(2):309-16. doi: 10.1097/SLA.0000000000002803

8. Towler B, Irwig L, Glasziou P, Kewenter J, Weller D, Silagy C. A systematic review of the effects of screening for colorectal cancer using the faecal occult blood test, hemoccult. Bmj (1998) 317(7158):559-65. doi: 10.1136/ bmj.317.7158.559

9. Chen LS, Yen AM, Fraser CG, Chiu SY, Fann JC, Wang PE, et al. Impact of faecal haemoglobin concentration on colorectal cancer mortality and allcause death. BMJ Open (2013) 3(11):e003740. doi: 10.1136/bmjopen-2013003740

10. Libby G, Fraser CG, Carey FA, Brewster DH, Steele RJC. Occult blood in faeces is associated with all-cause and non-colorectal cancer mortality. Gut (2018) 67(12):2116-23. doi: 10.1136/gutjnl-2018-316483

11. Xu BB, Lu J, Zheng ZF, Huang CM, Zheng CH, Xie JW, et al. Comparison of short-term and long-term efficacy of laparoscopic and open gastrectomy in high-risk patients with gastric cancer: a propensity score-matching analysis. Surg Endosc (2019) 33(1):58-70. doi: 10.1007/s00464-018-6268-Z

12. Fu Y, Wang L, Xie C, Zou K, Tu L, Yan W, et al. Comparison of non-invasive biomarkers faecal BAFF, calprotectin and FOBT in discriminating IBS from IBD and evaluation of intestinal inflammation. Sci Rep (2017) 7(1):2669. doi: 10.1038/s41598-017-02835-5

13. Liao Q, Zeng Z, Guo X, Li X, Wei F, Zhang W, et al. LPLUNC1 suppresses IL6-induced nasopharyngeal carcinoma cell proliferation via inhibiting the Stat3 activation. Oncogene (2014) 33(16):2098-109. doi: 10.1038/onc.2013.161

14. Zhou Y, Xia L, Liu Q, Wang H, Lin J, Oyang L, et al. Induction of ProInflammatory Response via Activated Macrophage-Mediated NF-kappaB and STAT3 Pathways in Gastric Cancer Cells. Cell Physiol Biochem (2018) 47 (4):1399-410. doi: 10.1159/000490829

15. Gupta M, Babic A, Beck AH, Terry K. TNF-alpha expression, risk factors, and inflammatory exposures in ovarian cancer: evidence for an inflammatory pathway of ovarian carcinogenesis? Hum Pathol (2016) 54:82-91. doi: 10.1016/j.humpath.2016.03.006

16. Dindo D, Demartines N, Clavien PA. Classification of surgical complications: a new proposal with evaluation in a cohort of 6336 patients and results of a survey. Ann Surg (2004) 240(2):205-13. doi: 10.1097/01.sla.0000133083. 54934.ae

17. Chen QY, Zhong Q, Liu ZY, Xie JW, Wang JB, Lin JX, et al. Does Noncompliance in Lymph Node Dissection Affect Oncological Efficacy in Gastric Cancer Patients Undergoing Radical Gastrectomy? Ann Surg Oncol (2019) 26(6):1759-71. doi: 10.1245/s10434-019-07217-x

18. Chen QY, Huang CM, Lin JX, Zheng CH, Li P, Xie JW, et al. Laparoscopic Infrapyloric Area Lymph Node Dissection with No. 14v Enlargement for Advanced Lower Gastric Cancer in Middle Colic Vein Approach. Ann Surg Oncol (2016) 23(3):951. doi: 10.1245/s10434-015-4992-3

19. Huang CM, Chen QY, Lin JX, Zheng CH, Li P, Xie JW, et al. Laparoscopic Suprapancreatic Lymph Node Dissection for Advanced Gastric Cancer Using a Left-Sided Approach. Ann Surg Oncol (2015) 22(7):2351. doi: 10.1245/ s10434-014-4309-y

20. Huang CM, Chen QY, Lin JX, Zheng CH, Li P, Xie JW, et al. Laparoscopic spleen-preserving no. 10 lymph node dissection for advanced proximal gastric cancer using a left approach. Ann Surg Oncol (2014) 21(6):2051. doi: 10.1245/ s10434-014-3492-1

21. Xu BB, Lu J, Zheng ZF, Xie JW, Wang JB, Lin JX, et al. The predictive value of the preoperative $\mathrm{C}$-reactive protein-albumin ratio for early recurrence and chemotherapy benefit in patients with gastric cancer after radical gastrectomy: using randomized phase III trial data. Gastric Cancer (2019) 22(5):1016-28. doi: 10.1007/s10120-019-00936-w

22. van der Vlugt M, Grobbee EJ, Bossuyt PM, Bos ACRK, Kuipers EJ, LansdorpVogelaar I, et al. Risk of Oral and Upper Gastrointestinal Cancers in Persons With Positive Results From a Fecal Immunochemical Test in a Colorectal Cancer Screening Program. Clin Gastroenterol Hepatol (2018) 16(8):123743.e2. doi: 10.1016/j.cgh.2018.01.037

23. He E, Alison R, Blanks R, Pirie K, Reeves G, Ward RL, et al. Association of ten gastrointestinal and other medical conditions with positivity to faecal occult blood testing in routine screening: a large prospective study of women in England. Int J Epidemiol (2019) 48(2):549-58. doi: 10.1093/ije/dyy271
24. Velez JP, Schwesinger WH, Stauffer J, Gaskill HV 3rd, Kazantsev GB, Sirinek $\mathrm{KR}$, et al. Bidirectional endoscopy in patients with fecal occult blood. Surg Endosc (2002) 16(1):117-20. doi: 10.1007/s004640080183

25. Barrows GH, Burton RM, Jarrett DD, Russell GG, Alford MD, Songster CL. Immunochemical detection of human blood in feces. Am J Clin Pathol (1978) 69(3):342-6. doi: 10.1093/ajcp/69.1.342

26. Wu TH, Lee TK, Yen MF, Tung TH, Chen TH. Long-term mortality assessment using biological measures among elderly people. Ten-year follow-up of 597 healthy elderly subjects in Taiwan. Fam Pract (2002) 19 (3):272-7. doi: 10.1093/fampra/19.3.272

27. Iwai N, Dohi O, Naito Y, Inada Y, Fukui A, Takayama S, et al. Impact of the Charlson comorbidity index and prognostic nutritional index on prognosis in patients with early gastric cancer after endoscopic submucosal dissection. Dig Endosc (2018) 30(5):616-23. doi: 10.1111/den.13051

28. Huang XZ, Yang YC, Chen Y, Wu CC, Lin RF, Wang ZN, et al. Preoperative Anemia or Low Hemoglobin Predicts Poor Prognosis in Gastric Cancer Patients: A Meta-Analysis. Dis Markers (2019) 2019:7606128. doi: 10.1155/ 2019/7606128

29. Onate-Ocana LF, Aiello-Crocifoglio V, Gallardo-Rincon D, Herrera-Goepfert R, Brom-Valladares R, Carrillo JF, et al. Serum albumin as a significant prognostic factor for patients with gastric carcinoma. Ann Surg Oncol (2007) 14(2):381-9. doi: 10.1245/s10434-006-9093-x

30. Balkwill F, Mantovani A. Inflammation and cancer: back to Virchow? Lancet (2001) 357(9255):539-45. doi: 10.1016/S0140-6736(00)04046-0

31. Chen L, Brar MS, Leung FC, Hsiao WL. Triterpenoid herbal saponins enhance beneficial bacteria, decrease sulfate-reducing bacteria, modulate inflammatory intestinal microenvironment and exert cancer preventive effects in ApcMin/+ mice. Oncotarget (2016) 7(21):31226-42. doi: 10.18632/oncotarget.8886

32. Su CY, Fu XL, Duan W, Yu PW, Zhao YL. High density of CD68+ tumorassociated macrophages predicts a poor prognosis in gastric cancer mediated by IL-6 expression. Oncol Lett (2018) 15(5):6217-24. doi: 10.3892/ ol.2018.8119

33. Weiss G, Goodnough LT. Anemia of chronic disease. N Engl J Med (2005) 352 (10):1011-23. doi: 10.1056/NEJMra041809

34. Li QG, Li P, Tang D, Chen J, Wang DR. Impact of postoperative complications on long-term survival after radical resection for gastric cancer. World J Gastroenterol (2013) 19(25):4060-5. doi: 10.3748/ wjg.v19.i25.4060

35. Sierzega M, Kolodziejczyk P, Kulig J, Polish Gastric Cancer Study G. Impact of anastomotic leakage on long-term survival after total gastrectomy for carcinoma of the stomach. Br J Surg (2010) 97(7):1035-42. doi: 10.1002/ bjs. 7038

36. Menetrier-Caux C, Montmain G, Dieu MC, Bain C, Favrot MC, Caux X, et al. Inhibition of the differentiation of dendritic cells from CD34(+) progenitors by tumor cells: role of interleukin- 6 and macrophage colony-stimulating factor. Blood (1998) 92(12):4778-91. doi: 10.1182/blood.V92.12.4778

37. Horn F, Henze C, Heidrich K. Interleukin-6 Signal Transduction and Lymphocyte Function. Immunobiology (2000) 202(2):151-67. doi: 10.1016/ S0171-2985(00)80061-3

38. Sun P, Xiang JB, Chen ZY. Meta-analysis of adjuvant chemotherapy after radical surgery for advanced gastric cancer. Br J Surg (2009) 96(1):26-33. doi: 10.1002/bjs.6408

39. Lv Y, Zhao Y, Wang X, Chen N, Mao F, Teng Y, et al. Increased intratumoral mast cells foster immune suppression and gastric cancer progression through TNF-alpha-PD-L1 pathway. J Immunother Cancer (2019) 7(1):54. doi: 10.1186/s40425-019-0530-3

40. Viitala MK, Virtakoivu R, Tadayon S, Rannikko J, Jalkanen S, Hollmen M. Immunotherapeutic Blockade of Macrophage Clever-1 Reactivates the CD8+ T Cell Response Against Immunosuppressive Tumors. Clin Cancer Res (2019) 25(11):3289-303. doi: 10.1158/1078-0432.CCR-18-3016

41. Ham IH, Oh HJ, Jin H, Bae CA, Jeon SM, Choi KS, et al. Targeting interleukin-6 as a strategy to overcome stroma-induced resistance to chemotherapy in gastric cancer. Mol Cancer (2019) 18(1):68. doi: 10.1186/s12943-019-0972-8

42. Elinav E, Nowarski R, Thaiss CA, Hu B, Jin C, Flavell RA. Inflammationinduced cancer: crosstalk between tumours, immune cells and microorganisms. Nat Rev Cancer (2013) 13(11):759-71. doi: 10.1038/nrc3611

43. Iida N, Dzutsev A, Stewart CA, Smith L, Bouladoux N, Weingarten RA, et al. Commensal bacteria control cancer response to therapy by modulating the 
tumor microenvironment. Science (2013) 342(6161):967-70. doi: 10.1126/ science. 1240527

44. Viaud S, Saccheri F, Mignot G, Yamazaki T, Daillere R, Hannani D, et al. The intestinal microbiota modulates the anticancer immune effects of cyclophosphamide. Science (2013) 342(6161):971-6. doi: 10.1126/ science. 1240537

45. Uchiyama K, Ida K, Okuda J, Asai Y, Ohyama Y, Kuroda M, et al. Correlations of hemoglobin index ( $\mathrm{IHb}$ ) of gastric mucosa with Helicobacter pylori (H. pylori) infection and inflammation of gastric mucosa. Scand J Gastroenterol (2004) 39(11):1054-60. doi: 10.1080/ 00365520410009645

46. Li W, Deng Y, Chu Q, Zhang P. Gut microbiome and cancer immunotherapy. Cancer Lett (2019) 447:41-7. doi: 10.1016/j.canlet.2019.01.015

47. Wang JT, Li H, Zhang H, Chen YF, Cao YF, Li RC, et al. Intratumoral IL17-producing cells infiltration correlate with antitumor immune contexture and improved response to adjuvant chemotherapy in gastric cancer. Ann Oncol (2019) 30(2):266-73. doi: 10.1093/annonc/mdy505
48. Jiang Y, Li T, Liang X, Hu Y, Huang L, Liao Z, et al. Association of Adjuvant Chemotherapy With Survival in Patients With Stage II or III Gastric Cancer. JAMA Surg (2017) 152(7):e171087. doi: 10.1001/jamasurg.2017.1087

Conflict of Interest: The authors declare that the research was conducted in the absence of any commercial or financial relationships that could be construed as a potential conflict of interest.

The handling Editor declared a past co-authorship with several of the authors JX, $\mathrm{CZ}, \mathrm{CH}, \mathrm{PL}$.

Copyright (C) $2021 \mathrm{Lu}, \mathrm{Xu}, \mathrm{Xu}$, Wu, Xie, Wang, Lin, Chen, Cao, Zheng, Huang and Li. This is an open-access article distributed under the terms of the Creative Commons Attribution License (CC BY). The use, distribution or reproduction in other forums is permitted, provided the original author(s) and the copyright owner(s) are credited and that the original publication in this journal is cited, in accordance with accepted academic practice. No use, distribution or reproduction is permitted which does not comply with these terms. 